\title{
Qualidade física do solo sob sistemas de preparo e cobertura morta em pomar de laranja
}

\author{
Jonez Fidalski( ${ }^{(1)}$, Graziela Moraes de Cesare Barbosa(2), Pedro Antonio Martins Auler ${ }^{(1)}$, \\ Marcos Antonio Pavan ${ }^{(2)}$ e José Marcos Garrido Beraldo ${ }^{(1)}$
} (1)Instituto Agronômico do Paraná (lapar), Caixa Postal 564, CEP 87701-970 Paranavaí, PR. E-mail: fidalski@iapar.br, aulerpe@iapar.br,
jmgberaldo@yahoo.com.br (2)Iapar, Caixa Postal 481, CEP 86001-970 Londrina, PR. E-mail: graziela_barbosa@iapar.br, mpavan@iapar.br

Resumo - O objetivo deste trabalho foi avaliar o efeito de preparo do solo e de cobertura morta sobre a qualidade física de um Latossolo, em um pomar de laranja 'Pêra'. O experimento foi instalado em Paranavaí, PR, em área com Brachiaria brizantha. O delineamento foi o de blocos ao acaso, em parcelas subsubdivididas, com 12 tratamentos e 4 repetições. Nas parcelas foram estudados os sistemas plantio direto, preparo em faixas e preparo convencional; nas subparcelas, os manejos com e sem cobertura morta, na linha das plantas de laranjeira 'Pêra'; e nas subsubparcelas, os porta-enxertos Citrus limonia Osb. e Citrus reshni Hort. ex Tan. Foram coletadas amostras de solo nas linhas das plantas, sob o rodado e no entrerrodado do trator, para quantificação de densidade, macroporosidade e microporosidade. O plantio direto de laranja em pastagem manteve a qualidade física do solo nas linhas das plantas, no entrerrodado e sob o rodado. O preparo convencional comprometeu a qualidade física do solo sob o rodado. A qualidade física do solo foi favorecida pelo menor revolvimento do solo, resultante do plantio direto ou do preparo em faixas, e pelo manejo da cobertura morta nas linhas das plantas, após o plantio das laranjeiras.

Termos para indexação: Brachiaria brizantha, citros, densidade do solo, manejo do solo, plantio direto, porosidade do solo.

\section{Soil physical quality under planting and mulching systems in an orange grove}

\begin{abstract}
The objective of this work was to evaluate the effects of planting and mulching systems on the soil physical quality of a Typic Haplorthox (Rhodic Ferralsol), in a grove cultivated with orange cultivar Pêra. The experiment was established in Paranavaí, PR, Brazil, in a field dominated by the forage grass Brachiaria brizantha. The experiment had a randomized complete block split-split plot design, with 12 treatments and 4 replicates. The main plots were no-tillage, strip-tillage, and conventional tillage systems; in the split plots, systems with or without mulching in plant rows were evaluated; and in the split-split plots, the root stocks Citrus limonia Osb. and Citrus reshni Hort. ex Tan. were evaluated. Soil samples were collected in the tree rows and under and in-between the tractor wheel tracks for soil bulk density, macroporosity, and microporosity. Orange planting with no-tillage maintained the soil physical quality in the tree rows and under and in-between the wheel tracks. Conventional tillage was detrimental to the physical quality of the soil under the wheel tracks. Soil physical quality was favored by the lowest soil disturbance in the establishment of the grove, both under no-tillage and strip-tillage, and it was also favored by mulching of Brachiaria brizantha in the tree rows, after planting.
\end{abstract}

Index terms: Brachiaria brizantha, citrus, soil bulk density, soil management, no-tillage, soil porosity.

\section{Introdução}

Os indicadores mais utilizados para medir a qualidade física do solo em pomares de laranja são a densidade, a macroporosidade, a microporosidade e a porosidade total (Sanches et al., 1999; Portela et al., 2001). Esses indicadores são influenciados pelos sistemas de manejo da cobertura permanente do solo, nas entrelinhas do pomar (Fidalski et al., 2007) e em pastagens (Fidalski et al., 2008b).
$\mathrm{Na}$ implantação de pomares de laranja, predomina o uso do preparo convencional, com revolvimento do solo e incorporação da cobertura vegetal em área total, o que acarreta erosão hídrica (Politano \& Pissarra, 2005). Durante a fase de formação e produção de laranja, a vegetação nas linhas e entrelinhas das plantas compete pela água do solo com as laranjeiras (Wright et al., 2003). Em experimentos realizados no noroeste do Paraná, a gramínea não comprometeu o

Pesq. agropec. bras., Brasília, v.44, n.1, p.76-83, jan. 2009 
desenvolvimento, a nutrição e a produção de laranja (Fidalski et al., 2007; Auler et al., 2008).

Em áreas de pastagens no noroeste do Paraná, o preparo em faixas para a implantação de pomar de laranja restringe a aração e gradagem a faixas de $2 \mathrm{~m}$ de largura, e preserva a gramínea nas entrelinhas de $5 \mathrm{~m}$ de largura (Auler et al., 2008). Este sistema, que favorece a fertilidade do solo, a nutrição das plantas e mantém a produção de laranja, reduz em $70 \%$ a área de preparo do solo. Essa redução pode ser ainda maior com o plantio direto de laranjeiras (Beraldo et al., 2007; Neves et al., 2007).

A vegetação nas entrelinhas do pomar de laranja pode ser roçada e movida para as linhas das plantas, para ser manejada como cobertura morta, o que pode aumentar a produção de laranja, a depender das espécies cultivadas nas entrelinhas (Beraldo et al., 2007; Bremer Neto et al., 2008).

Em pomares de laranja já formados e em fase de produção, tem sido verificada maior compactação do solo sob o rodado do trator do que no entrerrodado (Fidalski et al., 2007). No noroeste do Paraná, intervenções mecânicas nas entrelinhas de pomares em produção são consideradas inadequadas, por comprometer a qualidade física de solos desenvolvidos a partir do arenito da Formação Caiuá.

O objetivo deste trabalho foi avaliar o efeito de sistemas de preparo do solo e do manejo de cobertura morta sobre a qualidade física de um Latossolo Vermelho distrófico típico, em um pomar de laranja 'Pêra'.

\section{Material e Métodos}

A avaliação da qualidade física do solo foi realizada em um experimento de laranjeira 'Pêra' [Citrus sinensis (L.) Osb.], implantado em novembro de 2002, no espaçamento de 6,8x3,5 m, na Fazenda Ipiranga, da Cooperativa Cocamar, Município de Paranavaí, noroeste do Paraná $\left(23^{\circ} 6^{\prime} \mathrm{S}, 52^{\circ} 25^{\prime} \mathrm{W}\right.$ e altitude de $450 \mathrm{~m}$ ). O clima na região é subtropical (Cfa), com precipitações médias anuais de $1.500 \mathrm{~mm}$. O solo é um Latossolo Vermelho distrófico típico textura de arenosa/média A moderado, com relevo suaveondulado e declividade máxima de $6 \mathrm{~cm} \mathrm{~m}^{-1}$ (Santos et al., 2006), desenvolvido a partir do arenito da Formação Caiuá. O horizonte Amoderado foi verificado na profundidade de $0-20 \mathrm{~cm}\left(110 \mathrm{~g} \mathrm{~kg}^{-1}\right.$ de argila,
$15 \mathrm{~g} \mathrm{~kg}^{-1}$ de silte e $875 \mathrm{~g} \mathrm{~kg}^{-1}$ de areia), e o horizonte Bw, na profundidade de 20-200 cm (170 $\mathrm{g} \mathrm{kg}^{-1} \mathrm{de}$ argila, $10 \mathrm{~g} \mathrm{~kg}^{-1}$ de silte e $820 \mathrm{~g} \mathrm{~kg}^{-1}$ de areia).

$\mathrm{O}$ delineamento experimental foi o de blocos ao acaso, no esquema de parcela subsubdividida, com 12 tratamentos e 4 repetições. Na parcela, foram feitos três tipos de preparo do solo (plantio direto, preparo em faixas e preparo convencional); na subparcela, foram feitos dois manejos da cobertura do solo (sem e com cobertura morta, na linha das plantas de laranjeira 'Pêra'); e na subsubparcela, foram plantados dois portaenxertos: o limoeiro 'Cravo' (Citrus limonia Osb.) e a tangerineira 'Cleópatra' (Citrus reshni Hort. ex Tan.). Cada subsubparcela constituiu-se de 15 plantas, dispostas em três linhas com cinco plantas.

O preparo do solo foi realizado em novembro de 2002, em uma área anteriormente explorada com pastagem de Brachiaria brizantha (Hochst.) Stapf. Distribuíram-se 3,6 t ha ${ }^{-1}$ de calcário dolomítico sobre essa gramínea, para elevar a saturação por bases a 70\%. O plantio direto consistiu da dessecação da $B$. brizantha em faixas de $2 \mathrm{~m}$, destinadas às linhas das plantas, tendo-se mantido as entrelinhas vegetadas. $O$ preparo em faixas consistiu do preparo convencional de faixas de $2 \mathrm{~m}$ de largura, destinados às linhas das plantas, com a manutenção da B. brizantha nas entrelinhas (Auler et al., 2008). O preparo convencional consistiu de uma aração, a $20 \mathrm{~cm}$ de profundidade, e duas gradagens até $10 \mathrm{~cm}$ de profundidade. Em seguida ao preparo, foram abertos sulcos com $30 \mathrm{~cm}$ de profundidade, destinados à marcação das linhas de plantio.

O plantio das laranjeiras foi realizado em julho de 2003, e em dezembro de 2003 foi realizada uma roçada. A partir dessa data, foi realizado o corte da B. brizantha duas vezes ao ano, próximo ao início e ao final do período de maior precipitação (novembro e março), por meio de roçadora. Essa gramínea foi mantida nas entrelinhas (no tratamento em que a linha é mantida sem cobertura morta) ou deslocada para as linhas das plantas, sob a projeção da copa (no tratamento em que a linha é mantida com cobertura morta).

A invasão da $B$. brizantha em direção à linha das plantas foi controlada com glifosato, que a manteve até $50 \mathrm{~cm}$ distante da projeção das copas. O controle de plantas infestantes nas linhas das plantas foi efetuado por meio de capinas com enxada. 
A ocorrência de vegetação invasora, com presença de espécies de gramíneas e de folha larga, foi maior no tratamento sem cobertura morta. No tratamento com cobertura morta a presença de invasoras nas linhas das plantas caracterizou-se pela baixa ocorrência, de forma isolada e descontínua. De julho a dezembro de 2003, as capinas se restringiram à limpeza da coroa das plantas. A partir de 2004 até o final de 2006, foram realizadas, em média, quatro capinas por ano, em toda a extensão das linhas de plantio. O intervalo médio entre as capinas foi de três meses, o que permitiu o restabelecimento temporário da vegetação por invasoras, principalmente no tratamento sem cobertura morta nas linhas.

Amostras indeformadas de solo foram coletadas em dezembro de 2006, próximo à planta central de cada subsubparcela experimental, com anéis de aço com $5 \mathrm{~cm}$ de altura e $5 \mathrm{~cm}$ de diâmetro, nas camadas de 0-10, 10-20, 20-30 e 30-40 cm de profundidade. Essas amostras foram coletadas a 0,75 e 3,4 $\mathrm{m}$ de distância do tronco das laranjeiras, respectivamente, na linha das plantas e no centro da entrelinha (entrerrodado).

Em outubro de 2008, uma segunda amostragem de solo foi realizada nas entrelinhas, mediante a coleta de amostras de solo indeformadas na camada de 0-10 $\mathrm{cm}$ de profundidade, no entrerrodado e sob o rodado do trator. O rodado localiza-se entre as linhas das plantas e o centro das entrelinhas.
As amostras de solo foram submetidas às análises de macroporosidade, microporosidade, densidade do solo e densidade de partículas (Claessen, 1997). A macroporosidade foi obtida pela diferença entre o conteúdo de água saturado e o conteúdo de água após aplicação da tensão de $6 \mathrm{kPa}$. A microporosidade foi estimada como o conteúdo de água retido na tensão de $6 \mathrm{kPa}$. A densidade do solo foi calculada pela massa de solo seco a $105^{\circ} \mathrm{C}$ por 48 horas. A densidade de partículas do solo foi determinada com balão volumétrico e álcool etílico (Claessen, 1997).

Os resultados originais foram submetidos à análise de variância, por meio do modelo matemático de blocos ao acaso, no esquema de parcelas subsubdivididas, e as médias foram comparadas pelo teste de Tukey, a 5\% de probabilidade (Banzatto \& Kronka, 2006). Os indicadores de qualidade física do solo foram analisados individualmente, para cada camada, posição de amostragem e ano de amostragem de solo.

\section{Resultados e Discussão}

Nas linhas das plantas, não houve interação significativa entre os fatores sistemas de preparo do solo, sistemas de manejo da cobertura morta e porta-enxertos, em 2006 (Tabela 1). Nessa posição

Tabela 1. Probabilidades (teste F) obtidas das análises de variâncias dos indicadores de qualidade física do solo, para as causas de variação sistemas de preparo do solo (P), sistemas de manejo da cobertura morta (M) e porta-enxertos da laranjeira 'Pêra' (E), provenientes de amostras de solo coletadas em 2006, em quatro camadas nas linhas das plantas.

\begin{tabular}{|c|c|c|c|c|c|c|c|}
\hline Camada $(\mathrm{cm})$ & $\mathrm{P}$ & $\mathrm{M}$ & $\mathrm{E}$ & PxM & PxE & $\mathrm{MxE}$ & PxMxE \\
\hline & \multicolumn{7}{|c|}{ Densidade do solo } \\
\hline $0-10$ & 0,12 & 0,63 & 0,63 & 0,76 & 0,68 & 0,35 & 0,51 \\
\hline $10-20$ & 0,89 & 0,17 & 0,63 & 0,74 & 0,76 & 0,84 & 0,59 \\
\hline $20-30$ & 0,06 & 0,02 & 0,49 & 0,42 & 0,60 & 0,81 & 0,81 \\
\hline \multirow[t]{2}{*}{$30-40$} & 0,74 & 0,08 & 0,20 & 0,97 & 0,77 & 0,89 & 0,99 \\
\hline & \multicolumn{7}{|c|}{ Macroporosidade do solo } \\
\hline $0-10$ & 0,94 & 0,13 & 0,93 & 1,00 & 0,94 & 0,62 & 0,32 \\
\hline $10-20$ & 0,94 & 0,07 & 0,74 & 0,75 & 0,95 & 0,97 & 0,66 \\
\hline $20-30$ & 0,40 & 0,03 & 0,41 & 0,45 & 0,68 & 0,96 & 0,77 \\
\hline \multirow[t]{2}{*}{$30-40$} & 0,74 & 0,05 & 0,21 & 0,95 & 0,74 & 0,89 & 0,87 \\
\hline & \multicolumn{7}{|c|}{ Microporosidade do solo } \\
\hline $0-10$ & 0,21 & 0,06 & 0,30 & 0,99 & 0,63 & 0,39 & 0,25 \\
\hline $10-20$ & 0,65 & 0,03 & 0,27 & 0,58 & 0,73 & 0,93 & 0,99 \\
\hline $20-30$ & 0,72 & 0,06 & 0,40 & 0,57 & 0,78 & 0,97 & 0,81 \\
\hline \multirow[t]{2}{*}{$30-40$} & 0,74 & 0,07 & 0,31 & 0,82 & 0,75 & 0,49 & 0,66 \\
\hline & \multicolumn{7}{|c|}{ Porosidade total do solo } \\
\hline $0-10$ & 0,06 & 0,95 & 0,65 & 0,85 & 0,64 & 0,33 & 0,65 \\
\hline $10-20$ & 0,80 & 0,14 & 0,75 & 0,80 & 0,90 & 0,98 & 0,45 \\
\hline $20-30$ & 0,06 & 0,03 & 0,45 & 0,43 & 0,61 & 0,94 & 0,77 \\
\hline $30-40$ & 0,80 & 0,07 & 0,19 & 0,97 & 0,71 & 0,83 & 0,96 \\
\hline
\end{tabular}


de amostragem de solo, houve efeito isolado dos sistemas de manejo da cobertura morta na densidade do solo, macroporosidade e porosidade total, na camada de 20-30 cm, e na macroporosidade na camada de 30-40 cm. Sob o entrerrodado, houve interação significativa entre os fatores sistemas de preparo do solo, sistemas de manejo da cobertura morta e porta-enxertos, na camada de $0-10 \mathrm{~cm}$ quanto à macroporosidade em 2006 e à porosidade total em 2008 (Tabela 2). Outras interações significativas foram verificadas entre os sistemas de manejo da cobertura morta e os porta-enxertos quanto à macroporosidade $\mathrm{e}$ à microporosidade, e entre sistemas de preparo do solo e manejo da cobertura morta na camada de $0-10 \mathrm{~cm}$ em 2008. Efeito isolado dos sistemas de manejo da cobertura do solo foi verificado na microporosidade, nas camadas de $0-10,10-20,20-30$ e $30-40 \mathrm{~cm}$ em 2006. Em 2008, na camada de 0-10 cm, foram observados efeitos dos sistemas de preparo do solo na densidade do solo e porosidade total sob rodado e efeitos dos porta-enxertos na macroporosidade e porosidade total sob rodado e entrerrodado.

A densidade do solo, indicador mais utilizado na avaliação da qualidade física do solo, não foi influenciada, em 2006, pelos sistemas de preparo do solo nas linhas das plantas e no entrerrodado, nas camadas de $0-10,10-20,20-30$ e $30-40 \mathrm{~cm}$ (Tabelas 3 e 4). Marun \& Mella (1997) também verificaram que não houve alterações da densidade do solo, nas camadas de 0-10, 10-20, 20-30 e 30-40 cm, após dois anos com cultivos sucessivos de culturas anuais de verão e de inverno, com preparo convencional para culturas anuais, em solo similar ao deste trabalho.

Verificou-se diferença significativa entre os sistemas de preparo do solo, sob o rodado, na camada de 0-10 cm, em 2008 (Tabelas 4). O preparo convencional apresentou maior densidade do solo e menor porosidade total do que o preparo em faixas e o plantio direto. Isto significa que o não revolvimento

Tabela 2. Probabilidades (teste F) obtidas das análises de variâncias dos indicadores de qualidade física do solo, para as causas de variação sistemas de preparo do solo (P), sistemas de manejo da cobertura morta (M) e porta-enxertos da laranjeira 'Pêra' (E), provenientes de amostras de solo coletadas nas entrelinhas, em 2006 e 2008, em quatro camadas.

\begin{tabular}{|c|c|c|c|c|c|c|c|c|c|}
\hline $\begin{array}{l}\text { Posição de } \\
\text { amostragem }\end{array}$ & Ano & $\begin{array}{c}\text { Camada } \\
(\mathrm{cm})\end{array}$ & $\mathrm{P}$ & M & $\mathrm{E}$ & PxM & PxE & $\mathrm{MxE}$ & PxMxE \\
\hline & \multicolumn{9}{|c|}{ Densidade do solo } \\
\hline Entrerrodado & 2006 & $0-10$ & 0,21 & 0,73 & 0,14 & 0,49 & 0,52 & 0,60 & 0,20 \\
\hline Entrerrodado & 2006 & $10-20$ & 0,55 & 0,44 & 0,20 & 0,62 & 0,82 & 0,95 & 0,94 \\
\hline Entrerrodado & 2006 & $20-30$ & 0,65 & 0,77 & 0,15 & 0,45 & 0,29 & 0,89 & 0,37 \\
\hline Entrerrodado & 2006 & $30-40$ & 0,47 & 0,30 & 0,50 & 0,84 & 0,40 & 0,52 & 0,38 \\
\hline Entrerrodado & 2008 & $0-10$ & 0,10 & 0,90 & 0,42 & 0,88 & 0,26 & 0,61 & 0,07 \\
\hline \multirow[t]{2}{*}{$\underline{\text { Rodado }}$} & 2008 & $0-10$ & $<0,01$ & 0,99 & 0,18 & 0,61 & 0,05 & 0,34 & 0,08 \\
\hline & \multicolumn{9}{|c|}{ Macroporosidade do solo } \\
\hline Entrerrodado & 2006 & $0-10$ & 0,70 & 0,10 & 0,09 & 0,28 & 0,36 & 0,15 & 0,02 \\
\hline Entrerrodado & 2006 & $10-20$ & 0,48 & 0,08 & 0,40 & 0,98 & 0,60 & 0,92 & 0,58 \\
\hline Entrerrodado & 2006 & $20-30$ & 0,79 & 0,09 & 0,43 & 0,43 & 0,79 & 0,36 & 0,35 \\
\hline Entrerrodado & 2006 & $30-40$ & 0,22 & 0,08 & 0,56 & 0,86 & 0,72 & 0,65 & 0,53 \\
\hline Entrerrodado & 2008 & $0-10$ & 0,23 & 0,97 & 0,03 & 0,27 & 0,97 & 0,11 & 0,11 \\
\hline \multirow[t]{2}{*}{$\underline{\text { Rodado }}$} & 2008 & $0-10$ & 0,44 & 0,67 & $<0,01$ & 0,43 & 0,55 & $<0,01$ & 0,28 \\
\hline & \multicolumn{9}{|c|}{ Microporosidade do solo } \\
\hline Entrerrodado & 2006 & $0-10$ & 0,95 & $<0,01$ & 0,23 & 0,33 & 0,15 & 0,06 & 0,01 \\
\hline Entrerrodado & 2006 & $10-20$ & 0,31 & 0,02 & 0,87 & 0,95 & 0,55 & 0,79 & 0,39 \\
\hline Entrerrodado & 2006 & $20-30$ & 0,92 & $<0,01$ & 0,91 & 0,36 & 0,92 & 0,12 & 0,49 \\
\hline Entrerrodado & 2006 & $30-40$ & 0,18 & 0,05 & 0,57 & 0,67 & 0,97 & 0,81 & 0,66 \\
\hline Entrerrodado & 2008 & $0-10$ & 0,41 & 0,40 & 0,95 & 0,05 & 0,99 & 0,05 & 0,77 \\
\hline \multirow[t]{2}{*}{$\underline{\text { Rodado }}$} & 2008 & $0-10$ & 0,25 & 0,76 & 0,18 & 0,89 & 0,12 & 0,02 & 0,43 \\
\hline & \multicolumn{9}{|c|}{ Porosidade total do solo } \\
\hline Entrerrodado & 2006 & $0-10$ & 0,34 & 0,71 & 0,15 & 0,56 & 0,55 & 0,52 & 0,17 \\
\hline Entrerrodado & 2006 & $10-20$ & 0,36 & 0,47 & 0,23 & 0,79 & 0,78 & 1,00 & 0,88 \\
\hline Entrerrodado & 2006 & $20-30$ & 0,48 & 0,67 & 0,09 & 0,54 & 0,41 & 0,76 & 0,24 \\
\hline Entrerrodado & 2006 & $30-40$ & 0,35 & 0,27 & 0,49 & 0,79 & 0,36 & 0,55 & 0,37 \\
\hline Entrerrodado & 2008 & $0-10$ & 0,17 & 0,50 & 0,01 & 0,33 & 0,96 & 0,33 & 0,01 \\
\hline Rodado & 2008 & $0-10$ & $<0,01$ & 0,82 & 0,02 & 0,62 & 0,37 & 0,48 & 0,55 \\
\hline
\end{tabular}


Tabela 3. Valores médios, diferença mínima significativa (DMS) e coeficientes de variação (CV) dos indicadores de qualidade física do solo, obtidos de amostras de solo coletadas nas linhas das plantas em 2006, em quatro camadas, para os tratamentos sistemas de preparo do solo plantio direto (PD), preparo em faixas (PF) e plantio convencional (PC) ${ }^{(1)}$.

\begin{tabular}{|c|c|c|c|c|c|}
\hline \multirow{2}{*}{\multicolumn{6}{|c|}{$\frac{\mathrm{PC}}{\text { Densidade do solo }\left(\mathrm{Mg} \mathrm{m}^{-3}\right)}$}} \\
\hline & & & & & \\
\hline $0-10$ & $1,58 \mathrm{a}$ & $1,57 \mathrm{a}$ & $1,60 \mathrm{a}$ & 0,05 & 2,64 \\
\hline $10-20$ & $1,56 \mathrm{a}$ & $1,56 \mathrm{a}$ & $1,57 \mathrm{a}$ & 0,12 & 7,00 \\
\hline $20-30$ & $1,54 \mathrm{a}$ & $1,57 \mathrm{a}$ & $1,59 \mathrm{a}$ & 0,06 & 3,40 \\
\hline $30-40$ & $1,51 \mathrm{a}$ & $1,52 \mathrm{a}$ & $1,54 \mathrm{a}$ & 0,11 & 6,81 \\
\hline \multicolumn{6}{|c|}{ Macroporosidade do solo $\left(\mathrm{m}^{3} \mathrm{~m}^{-3}\right)$} \\
\hline $0-10$ & $0,12 \mathrm{a}$ & $0,12 \mathrm{a}$ & $0,13 \mathrm{a}$ & 0,04 & 27,63 \\
\hline $10-20$ & $0,12 \mathrm{a}$ & $0,13 \mathrm{a}$ & $0,13 \mathrm{a}$ & 0,08 & 61,87 \\
\hline $20-30$ & $0,14 \mathrm{a}$ & $0,13 \mathrm{a}$ & $0,11 \mathrm{a}$ & 0,06 & 42,04 \\
\hline $30-40$ & $0,19 \mathrm{a}$ & $0,18 \mathrm{a}$ & $0,17 \mathrm{a}$ & 0,07 & 36,38 \\
\hline \multicolumn{6}{|c|}{ Microporosidade do solo $\left(\mathrm{m}^{3} \mathrm{~m}^{-3}\right)$} \\
\hline $0-10$ & $0,28 \mathrm{a}$ & $0,28 \mathrm{a}$ & $0,26 \mathrm{a}$ & 0,03 & 9,66 \\
\hline $10-20$ & $0,29 \mathrm{a}$ & $0,28 \mathrm{a}$ & $0,28 \mathrm{a}$ & 0,05 & 15,72 \\
\hline $20-30$ & $0,28 \mathrm{a}$ & $0,27 \mathrm{a}$ & $0,29 \mathrm{a}$ & 0,04 & 12,78 \\
\hline $30-40$ & $0,24 \mathrm{a}$ & $0,24 \mathrm{a}$ & $0,25 \mathrm{a}$ & 0,03 & 12,40 \\
\hline \multicolumn{6}{|c|}{ Porosidade total do solo $\left(\mathrm{m}^{3} \mathrm{~m}^{-3}\right)$} \\
\hline $0-10$ & $0,40 \mathrm{a}$ & $0,40 \mathrm{a}$ & $0,39 \mathrm{a}$ & 0,02 & 3,87 \\
\hline $10-20$ & $0,41 \mathrm{a}$ & $0,41 \mathrm{a}$ & $0,40 \mathrm{a}$ & 0,05 & 11,56 \\
\hline $20-30$ & $0,42 \mathrm{a}$ & $0,40 \mathrm{a}$ & $0,39 \mathrm{a}$ & 0,04 & 4,68 \\
\hline $30-40$ & $0,42 \mathrm{a}$ & $0,42 \mathrm{a}$ & $0,42 \mathrm{a}$ & 0,04 & 9,47 \\
\hline
\end{tabular}

${ }^{(1)}$ Médias seguidas de letras iguais, nas linhas, não diferem entre si, a 5\% de probabilidade, pelo teste de Tukey.

Tabela 4. Valores médios, diferença mínima significativa (DMS) e coeficientes de variação (CV) dos indicadores de qualidade física do solo, obtidos de amostras coletadas nas entrelinhas (rodado e entrerrodado), em 2006 e 2008, em quatro camadas, para os tratamentos sistemas de preparo do solo plantio direto (PD), preparo em faixas (PF) e plantio convencional (PC) $)^{(1)}$.

\begin{tabular}{|c|c|c|c|c|c|c|c|}
\hline Posição de amostragem & Ano & Camada $(\mathrm{cm})$ & PD & $\mathrm{PF}$ & $\mathrm{PC}$ & DMS & CV $(\%)$ \\
\hline & \multicolumn{7}{|c|}{ Densidade do solo $\left(\mathrm{Mg} \mathrm{m}^{-3}\right)$} \\
\hline Entrerrodado & 2006 & $0-10$ & $1,64 \mathrm{a}$ & $1,63 \mathrm{a}$ & $1,65 \mathrm{a}$ & 0,03 & 1,44 \\
\hline Entrerrodado & 2006 & $10-20$ & $1,64 \mathrm{a}$ & $1,63 a$ & $1,64 \mathrm{a}$ & 0,05 & 2,83 \\
\hline Entrerrodado & 2006 & $20-30$ & $1,62 \mathrm{a}$ & $1,61 \mathrm{a}$ & $1,63 \mathrm{a}$ & 0,08 & 4,33 \\
\hline Entrerrodado & 2006 & $30-40$ & $1,58 \mathrm{a}$ & $1,57 \mathrm{a}$ & $1,60 \mathrm{a}$ & 0,08 & 4,75 \\
\hline Entrerrodado & 2008 & $0-10$ & $1,51 \mathrm{a}$ & $1,54 \mathrm{a}$ & $1,58 \mathrm{a}$ & 0,08 & 4,69 \\
\hline \multirow[t]{2}{*}{ Rodado } & 2008 & $0-10$ & $1,58 \mathrm{~b}$ & $1,59 \mathrm{~b}$ & $1,64 \mathrm{a}$ & 0,04 & 2,08 \\
\hline & \multicolumn{7}{|c|}{ Macroporosidade do solo $\left(\mathrm{m}^{3} \mathrm{~m}^{-3}\right)$} \\
\hline Entrerrodado & 2006 & $0-10$ & $0,10 \mathrm{a}$ & $0,11 \mathrm{a}$ & $0,10 \mathrm{a}$ & 0,04 & 35,16 \\
\hline Entrerrodado & 2006 & $10-20$ & $0,08 \mathrm{a}$ & $0,09 \mathrm{a}$ & $0,09 \mathrm{a}$ & 0,04 & 45,68 \\
\hline Entrerrodado & 2006 & $20-30$ & $0,11 \mathrm{a}$ & $0,11 \mathrm{a}$ & $0,10 \mathrm{a}$ & 0,06 & 51,73 \\
\hline Entrerrodado & 2006 & $30-40$ & $0,15 \mathrm{a}$ & $0,16 a$ & $0,13 a$ & 0,05 & 26,07 \\
\hline Entrerrodado & 2008 & $0-10$ & $0,14 \mathrm{a}$ & $0,12 \mathrm{a}$ & $0,12 \mathrm{a}$ & 0,05 & 33,63 \\
\hline \multirow[t]{2}{*}{ Rodado } & 2008 & $0-10$ & $0,09 \mathrm{a}$ & $0,09 \mathrm{a}$ & $0,07 \mathrm{a}$ & 0,03 & 36,44 \\
\hline & \multicolumn{7}{|c|}{ Microporosidade do solo $\left(\mathrm{m}^{3} \mathrm{~m}^{-3}\right)$} \\
\hline Entrerrodado & 2006 & $0-10$ & $0,27 \mathrm{a}$ & $0,27 \mathrm{a}$ & $0,28 \mathrm{a}$ & 0,04 & 12,52 \\
\hline Entrerrodado & 2006 & $10-20$ & $0,30 \mathrm{a}$ & $0,29 \mathrm{a}$ & $0,29 a$ & 0,03 & 7,84 \\
\hline Entrerrodado & 2006 & $20-30$ & $0,28 \mathrm{a}$ & $0,28 \mathrm{a}$ & $0,29 \mathrm{a}$ & 0,03 & 10,67 \\
\hline Entrerrodado & 2006 & $30-40$ & $0,26 \mathrm{a}$ & $0,25 \mathrm{a}$ & $0,26 \mathrm{a}$ & 0,02 & 8,07 \\
\hline Entrerrodado & 2008 & $0-10$ & $0,27 \mathrm{a}$ & $0,29 a$ & $0,28 \mathrm{a}$ & 0,03 & 11,41 \\
\hline \multirow[t]{2}{*}{ Rodado } & 2008 & $0-10$ & $0,30 \mathrm{a}$ & $0,29 \mathrm{a}$ & $0,28 \mathrm{a}$ & 0,02 & 7,10 \\
\hline & \multicolumn{7}{|c|}{ Porosidade total do solo $\left(\mathrm{m}^{3} \mathrm{~m}^{-3}\right)$} \\
\hline Entrerrodado & 2006 & $0-10$ & $0,38 \mathrm{a}$ & $0,38 \mathrm{a}$ & $0,37 \mathrm{a}$ & 0,01 & 3,19 \\
\hline Entrerrodado & 2006 & $10-20$ & $0,38 \mathrm{a}$ & $0,39 \mathrm{a}$ & $0,38 \mathrm{a}$ & 0,02 & 4,80 \\
\hline Entrerrodado & 2006 & $20-30$ & $0,39 \mathrm{a}$ & $0,39 a$ & $0,38 \mathrm{a}$ & 0,03 & 7,65 \\
\hline Entrerrodado & 2006 & $30-40$ & $0,40 \mathrm{a}$ & $0,41 \mathrm{a}$ & $0,39 a$ & 0,03 & 6,90 \\
\hline Entrerrodado & 2008 & $0-10$ & $0,41 \mathrm{a}$ & $0,41 \mathrm{a}$ & $0,39 \mathrm{a}$ & 0,03 & 7,11 \\
\hline Rodado & 2008 & $0-10$ & $0,38 \mathrm{a}$ & $0,37 \mathrm{a}$ & $0,36 \mathrm{~b}$ & 0,01 & 2,93 \\
\hline
\end{tabular}

${ }^{(1)}$ Médias seguidas de letras iguais, nas linhas, não diferem entre si, a 5\% de probabilidade, pelo teste de Tukey. 
do solo nas entrelinhas, utilizado no preparo em faixas e no plantio direto, assegurou melhor qualidade física na camada superficial arenosa desse solo. Machado et al. (2008) descreveram a degradação dessa classe de solo, com o preparo convencional do solo para culturas anuais, em condições climáticas semelhantes às do presente trabalho.

Com base nos resultados dos indicadores de qualidade física do solo (Tabelas 3 e 4), não havia limitações físicas do solo, como camadas compactadas que poderiam ser identificadas pela densidade do solo e porosidade total, que justificassem o preparo do Latossolo Vermelho distrófico típico para o plantio de laranjeiras, na área de pastagem com B. brizantha. Portanto, nas condições edafoclimáticas do noroeste do Paraná, não se esperam limitações físicas do solo ao plantio de laranjeira no sistema plantio direto e preparo em faixas, tendo-se como vantagens: a redução da erosão hídrica, em razão da cobertura do solo em área de pastagens, na fase de formação do pomar; e a redução do custo com operações mecanizadas para a implantação do pomar (Politano \& Pissarra, 2005; Neves et al., 2007; Auler et al., 2008).

A implantação de pomares de laranja em plantio direto, nesses solos, é viabilizada tecnicamente com a comprovação da eficiência da calagem superficial na correção do solo, a $20 \mathrm{~cm}$ de profundidade - nas condições edafoclimáticas do noroeste do Paraná -, em solos desenvolvidos a partir do arenito da Formação Caiuá (Fidalski \& Auler, 2008). O calcário deve ser distribuído na superfície do solo de todo o pomar, antes do plantio das mudas de laranjeiras, e a calagem deve ser repetida durante o desenvolvimento e produção das laranjeiras.

A cobertura morta de B. brizantha, nas linhas das plantas, aumentou a microporosidade na camada de 10-20 cm (Tabelas 5 e 6), e a remoção dessa gramínea das entrelinhas aumentou a microporosidade no entrerrodado, nas camadas de 0-10, 10-20, 20-30 e 30-40 cm (Tabela 6). A remoção da braquiária das entrelinhas para as linhas das plantas assegurou melhor homogeneidade da qualidade física do solo, entre essas duas posições de amostragem. Esses dados concordam com os de Fidalski et al. (2008a), que fizeram manejo de solo similar com Paspalum notatum e destacaram que a microporosidade desses solos é importante para o armazenamento de água em horizontes superficiais de textura arenosa.
O maior benefício do uso da cobertura morta de B. brizantha nas linhas do pomar, a partir da roçada e do deslocamento da biomassa produzida nas entrelinhas, se deve à maior microporosidade verificada em todo o perfil do solo, com diferenças significativas na camada de $10-20 \mathrm{~cm}$ nas linhas das plantas, e em todas as camadas, no entrerrodado (Tabelas 5 e 6). A maior microporosidade não se deve ao teor de carbono orgânico do solo nas linhas das plantas e no entrerrodado, que se manteve homogêneo entre essas duas posições, mas pode estar relacionada ao menor desenvolvimento das raízes da $B$. brizantha nas entrelinhas, com as sucessivas roçadas (Beraldo et al., 2007). Isto provocou redução da fertilidade na camada de $0-20 \mathrm{~cm}$, da altura das plantas e da produção de matéria verde. De modo similar, a cobertura morta reduziu a quantidade de raízes de plantas invasoras nas linhas da plantas. Fidalski \& Tormena (2007) verificaram aumento da macroporosidade, pelo efeito biológico das raízes

Tabela 5. Valores médios, diferença mínima significativa (DMS) e coeficientes de variação (CV) dos indicadores de qualidade física do solo, obtidos de amostras coletadas nas linhas das laranjeiras 'Pêra', em 2006, em quatro camadas, para os tratamentos sem cobertura morta nas linhas das plantas (SC) e com cobertura morta nas linhas das plantas $(\mathrm{CC})^{(1)}$.

\begin{tabular}{|c|c|c|c|c|}
\hline $\begin{array}{l}\text { Camada } \\
(\mathrm{cm})\end{array}$ & $\mathrm{SC}$ & $\mathrm{CC}$ & DMS & $\mathrm{CV}(\%)$ \\
\hline & \multicolumn{4}{|c|}{ Densidade do solo $\left(\mathrm{Mg} \mathrm{m}^{-3}\right)$} \\
\hline $0-10$ & $1,59 \mathrm{a}$ & $1,58 \mathrm{a}$ & 0,04 & 4,20 \\
\hline $10-20$ & $1,54 \mathrm{a}$ & $1,59 \mathrm{a}$ & 0,07 & 7,28 \\
\hline $20-30$ & $1,55 b$ & $1,59 \mathrm{a}$ & 0,04 & 3,48 \\
\hline \multirow[t]{2}{*}{$30-40$} & $1,50 \mathrm{a}$ & $1,55 \mathrm{a}$ & 0,06 & 6,51 \\
\hline & \multicolumn{4}{|c|}{ Macroporosidade do solo $\left(\mathrm{m}^{3} \mathrm{~m}^{-3}\right)$} \\
\hline $0-10$ & $0,14 \mathrm{a}$ & $0,11 \mathrm{a}$ & 0,04 & 48,11 \\
\hline $10-20$ & $0,15 \mathrm{a}$ & $0,10 \mathrm{a}$ & 0,06 & 73,38 \\
\hline $20-30$ & $0,15 \mathrm{a}$ & $0,10 \mathrm{~b}$ & 0,04 & 49,51 \\
\hline \multirow[t]{2}{*}{$\underline{30-40}$} & $0,20 \mathrm{a}$ & $0,15 \mathrm{a}$ & 0,05 & 41,86 \\
\hline & \multicolumn{4}{|c|}{ Microporosidade do solo $\left(\mathrm{m}^{3} \mathrm{~m}^{-3}\right)$} \\
\hline $0-10$ & $0,26 \mathrm{a}$ & $0,29 \mathrm{a}$ & 0,04 & 16,84 \\
\hline $10-20$ & $0,27 \mathrm{~b}$ & $0,30 \mathrm{a}$ & 0,03 & 16,84 \\
\hline $20-30$ & $0,27 \mathrm{a}$ & $0,29 \mathrm{a}$ & 0,03 & 15,58 \\
\hline \multirow[t]{2}{*}{ 30-40 } & $0,23 \mathrm{a}$ & $0,26 \mathrm{a}$ & 0,03 & 18,39 \\
\hline & \multicolumn{4}{|c|}{ Porosidade total do solo $\left(\mathrm{m}^{3} \mathrm{~m}^{-3}\right)$} \\
\hline $0-10$ & $0,40 \mathrm{a}$ & $0,40 \mathrm{a}$ & 0,02 & 6,25 \\
\hline $10-20$ & $0,42 \mathrm{a}$ & $0,40 \mathrm{a}$ & 0,03 & 11,24 \\
\hline $20-30$ & $0,41 \mathrm{a}$ & $0,40 \mathrm{~b}$ & 0,01 & 5,13 \\
\hline $30-40$ & $0,43 \mathrm{a}$ & $0,41 \mathrm{a}$ & 0,03 & 8,59 \\
\hline
\end{tabular}

${ }^{(1)}$ Médias seguidas de letras iguais, nas linhas, não diferem entre si, a $5 \%$ de probabilidade, pelo teste de Tukey. 
Tabela 6. Valores médios, diferença mínima significativa (DMS) e coeficientes de variação $(\mathrm{CV})$ dos indicadores de qualidade física do solo, obtidos de amostras coletadas nas entrelinhas (rodado e entrerrodado) em 2006 e 2008, em quatro camadas, para os tratamentos sem cobertura morta nas linhas das plantas (SC) e com cobertura morta nas linhas das plantas $(\mathrm{CC})^{(1)}$.

\begin{tabular}{|c|c|c|c|c|c|c|}
\hline $\begin{array}{l}\text { Posição de } \\
\text { amostragem }\end{array}$ & Ano & $\begin{array}{c}\text { Camada } \\
(\mathrm{cm})\end{array}$ & $\mathrm{SC}$ & $\mathrm{CC}$ & DMS & $\begin{array}{l}\text { CV } \\
(\%) \\
\end{array}$ \\
\hline \multicolumn{7}{|c|}{ Densidade do solo $\left(\mathrm{Mg} \mathrm{m}^{-3}\right)$} \\
\hline Entrerrodado & 2006 & $0-10$ & $1,64 \mathrm{a}$ & $1,64 \mathrm{a}$ & 0,04 & 4,13 \\
\hline Entrerrodado & 2006 & $10-20$ & $1,63 a$ & $1,64 \mathrm{a}$ & 0,05 & 4,45 \\
\hline Entrerrodado & 2006 & $20-30$ & $1,61 \mathrm{a}$ & $1,62 \mathrm{a}$ & 0,06 & 5,94 \\
\hline Entrerrodado & 2006 & $30-40$ & $1,57 \mathrm{a}$ & $1,60 \mathrm{a}$ & 0,07 & 6,76 \\
\hline Entrerrodado & 2008 & $0-10$ & $1,54 \mathrm{a}$ & $1,55 \mathrm{a}$ & 0,05 & 4,43 \\
\hline Rodado & 2008 & $0-10$ & $1,61 \mathrm{a}$ & $1,61 \mathrm{a}$ & 0,03 & 2,94 \\
\hline \multicolumn{7}{|c|}{ Macroporosidade do solo $\left(\mathrm{m}^{3} \mathrm{~m}^{-3}\right)$} \\
\hline Entrerrodado & 2006 & $0-10$ & $0,12 \mathrm{a}$ & $0,09 \mathrm{a}$ & 0,03 & 44,44 \\
\hline Entrerrodado & 2006 & $10-20$ & $0,10 \mathrm{a}$ & $0,07 \mathrm{a}$ & 0,03 & 60,67 \\
\hline Entrerrodado & 2006 & $20-30$ & $0,12 \mathrm{a}$ & $0,09 \mathrm{a}$ & 0,05 & 65,58 \\
\hline Entrerrodado & 2006 & $30-40$ & $0,17 \mathrm{a}$ & $0,12 \mathrm{a}$ & 0,05 & 53,26 \\
\hline odado & 2008 & $0-10$ & $0,12 \mathrm{a}$ & $0,12 \mathrm{a}$ & 0,02 & 22,62 \\
\hline Rodado & 2008 & $0-10$ & $0,08 \mathrm{a}$ & $0,08 \mathrm{a}$ & 0,02 & 32,27 \\
\hline \multicolumn{7}{|c|}{ Microporosidade do solo $\left(\mathrm{m}^{3} \mathrm{~m}^{-3}\right)$} \\
\hline Entrerrodado & 2006 & $0-10$ & $0,26 \mathrm{~b}$ & $0,29 \mathrm{a}$ & 0,02 & 9,83 \\
\hline Entrerrodado & 2006 & $10-20$ & $0,28 \mathrm{~b}$ & $0,31 \mathrm{a}$ & 0,02 & 9,91 \\
\hline Entrerrodado & 2006 & $20-30$ & $0,27 \mathrm{~b}$ & $0,30 \mathrm{a}$ & 0,02 & 12,50 \\
\hline Entrerrodado & 2006 & $30-40$ & $0,24 \mathrm{~b}$ & $0,27 \mathrm{a}$ & 0,03 & 13,52 \\
\hline Entrerrodado & 2008 & $0-10$ & $0,28 \mathrm{a}$ & $0,27 \mathrm{a}$ & 0,03 & 14,13 \\
\hline Rodado & 2008 & $0-10$ & $0,29 \mathrm{a}$ & $0,29 \mathrm{a}$ & 0,01 & 6,04 \\
\hline \multicolumn{7}{|c|}{ Porosidade total do solo $\left(\mathrm{m}^{3} \mathrm{~m}^{-3}\right)$} \\
\hline Entrerr & 2006 & $0-10$ & $0,38 \mathrm{a}$ & $0,38 \mathrm{a}$ & 0,02 & 7,00 \\
\hline Entrerrodado & 2006 & $10-20$ & $0,38 \mathrm{a}$ & $0,38 \mathrm{a}$ & 0,02 & 8,11 \\
\hline Entrerrodado & 2006 & $20-30$ & $0,39 \mathrm{a}$ & $0,39 \mathrm{a}$ & 0,02 & 9,16 \\
\hline Entrerrodado & 2006 & $30-40$ & $0,41 \mathrm{a}$ & $0,39 \mathrm{a}$ & 0,03 & 9,76 \\
\hline Entrerrodado & 2008 & $0-10$ & $0,41 \mathrm{a}$ & $0,40 \mathrm{a}$ & 0,03 & 11,92 \\
\hline Rodado & 2008 & $0-10$ & $0,37 \mathrm{a}$ & $0,37 \mathrm{a}$ & 0,02 & 7,39 \\
\hline
\end{tabular}

${ }^{(1)}$ Médias seguidas de letras iguais, nas linhas, não diferem entre si, a 5\% de probabilidade, pelo teste de Tukey.

do amendoim forrageiro (Arachis pintoi) em solo similar ao do presente trabalho.

Nas linhas das plantas, o manejo da cobertura morta com $B$. brizantha foi mais importante do que os sistemas de preparo do solo, para diferenciar a qualidade física do solo, expressa pela maior microporosidade na camada de 10-20 cm (Tabela 5). Tais resultados estão de acordo com os trabalhos de preparo do solo e manejo da cobertura permanente das entrelinhas de pomar de laranja, com a grama batatais (Paspalum notatum), em solo e clima semelhantes (Fidalski et al., 2007).

Nas entrelinhas do pomar, não houve efeito dos sistemas de preparo na qualidade física do solo no entrerrodado, em 2006 e 2008; mas, sob o rodado, o preparo em faixas e o plantio direto proporcionaram melhor qualidade física ao solo, em comparação ao preparo convencional, em 2008 (Tabela 4).
Tais resultados corroboram a eficiência do preparo em faixas e do plantio direto, com a vantagem econômica de reduzir as operações mecânicas de preparo do solo em pelo menos 70\% (Auler et al., 2008), além de reduzir os riscos da erosão hídrica na fase de implantação dos pomares de laranja, em solo com o horizonte superficial com textura arenosa (Fidalski \& Auler, 1997).

\section{Conclusões}

1. O plantio direto de laranja, em pastagem de Brachiaria brizantha, em Latossolo Vermelho distrófico típico, não compromete a qualidade física do solo nas linhas das plantas, no entrerrodado e sob o rodado do trator.

2. O preparo convencional compromete a qualidade física do solo sob o rodado.

3. A qualidade física do solo é influenciada pelo menor revolvimento do solo, resultante do plantio direto ou do preparo em faixas, e pelo manejo da cobertura morta de Brachiaria brizantha nas linhas das plantas, após o plantio das laranjeiras.

\section{Agradecimentos}

À Cocamar Cooperativa Agroindustrial, pela cessão da área experimental e apoio financeiro.

\section{Referências}

AULER, P.A.M.; FIDALSKI, J.; PAVAN, M.A.; NEVES, C.S.V.J. Produção de laranja 'Pêra' em sistemas de preparo de solo e manejo nas entrelinhas. Revista Brasileira de Ciência do Solo, v.32, p.363-374, 2008.

BANZATTO, D.A.; KRONKA, S. do N. Experimentação agrícola. 4.ed. Jaboticabal: Funep, 2006. 237p.

BERALDO, J.M.G.; AULER, P.A.M.; PAVAN, M.A.; FIDALSKI, J. Reciclagem de nutrientes num pomar de laranjeira 'Pêra' com diferentes sistemas de preparo de solo e cobertura vegetal. In: CONGRESSO BRASILEIRO DE CIÊNCIA DO SOLO, 31., 2007, Gramado. Conquistas e desafios da ciência do solo brasileira: anais. Viçosa: Sociedade Brasileira de Ciência do Solo, 2007. 1 CD-ROM.

BREMER NETO, H.; VICTORIA FILHO, R.; MOURÃO FILHO, F. de A.A.; MENEZES, G.M. de; CANALI, É. Estado nutricional e produção de laranjeira 'Pêra' em função da vegetação intercalar e cobertura morta. Pesquisa Agropecuária Brasileira, v.43, p.29-35, 2008.

CLAESSEN, M.E.E (Org.). Manual de métodos de análise de solo. 2.ed. rev. atual. Rio de Janeiro: Embrapa-CNPS, 1997. 212p. (Embrapa-CNPS. Documentos, 1). 
FIDALSKI, J.; AULER, P.A.M. Alterações químicas temporais nas faixas de adubação e entrelinhas do pomar, nutrição e produção de laranja após calagem superficial. Revista Brasileira de Ciência do Solo, v.32, p.689-696, 2008.

FIDALSKI, J.; AULER, P.A.M. Levantamento nutricional de pomares de laranja no noroeste do Paraná. Arquivos de Biologia e Tecnologia, v.40, p.443-451, 1997.

FIDALSKI, J.; MARUR, C.J.; TORMENA, C.A. Respostas fisiológicas da laranjeira 'Pêra' aos sistemas de manejo de cobertura permanente do solo nas entrelinhas. Revista Brasileira de Ciência do Solo, v.32, p.1307-1317, 2008a.

FIDALSKI, J.; TORMENA, C.A. Homogeneidade da qualidade física do solo nas entrelinhas de um pomar de laranjeira com sistemas de manejo da vegetação permanente. Revista Brasileira de Ciência do Solo, v.31, p.637-645, 2007.

FIDALSKI, J.; TORMENA, C.A.; CECATO, U.; BARBERO, L.M.; LUGÃO, S.M.B.; COSTA, M.A.T. Qualidade física do solo em pastagem adubada e sob pastejo contínuo. Pesquisa Agropecuária Brasileira, v.43, p.1583-1590, $2008 \mathrm{~b}$.

FIDALSKI, J.; TORMENA, C.A.; SILVA, A.P. da. Qualidade física do solo em pomar de laranjeira no noroeste do Paraná com manejo da cobertura permanente na entrelinha. Revista Brasileira de Ciência do Solo, v.31, p.423-433, 2007.

MACHADO, J.L.; TORMENA, C.A.; FIDALSKI, J.; SCAPIM, C.A. Inter-relações entre as propriedades físicas e os coeficientes da curva de retenção de água de um Latossolo sob diferentes sistemas de uso. Revista Brasileira de Ciência do Solo, v.32, p.495-502, 2008.

MARUN, F.; MELLA, S.C. Recuperação de pastagens pela sucessão de culturas anuais e bianuais: um estudo no noroeste do Paraná. Londrina: Iapar, 1997. 24p. (Iapar. Boletim Técnico, $52)$.

NEVES, C.S.V.J.; TAVARES FILHO, J.; BRITO, O.R.; YAMASHITA, F.; TORMEM, V. Producción y desarrollo de las plantas y características físicas y químicas del suelo, en un huerto de cítricos plantado con dos sistemas de labranza en un Oxisol brasileño. In: SIMPOSIO INTERNACIONAL DE FRUTICULTURA TROPICAL Y SUBTROPICAL, 2., 2007, Habana. Memorias. Habana: IIFT, 2007.

POLITANO, W.; PISSARRA, T.C.T. Avaliação por fotointerpretação das áreas de abrangência dos diferentes estados da erosão acelerada do solo em canaviais e pomares de citros. Engenharia Agrícola, v.25, p.242-252, 2005.

PORTELA, J.C.; LIBARDI, P.L.; LIER, Q. de J. van. Retenção da água em solo sob diferentes usos no ecossistema Tabuleiros Costeiros. Revista Brasileira de Engenharia Agrícola e Ambiental, v.5, p.49-54, 2001.

SANCHES, A.C.; SILVA, A.P.; TORMENA, C.A.; RIGOLIN, A.T. Impacto do cultivo de citros em propriedades químicas, densidade do solo e atividade microbiana de um Podzólico Vermelho-Amarelo. Revista Brasileira de Ciência do Solo, v.23, p.91-99, 1999.

SANTOS, H.G. dos; JACOMINE, P.K.T.; ANJOS, L.H.C. dos; OLIVEIRA, V.A. de; OLIVEIRA, J.B. de; COELHO, M.R.; LUMBRERAS, J.F.; CUNHA, T.J.F. (Ed.). Sistema brasileiro de classificação de solos. 2.ed. Rio de Janeiro: Embrapa Solos, 2006. $306 \mathrm{p}$.

WRIGHT, G.C.; McCLOSKEY, W.B.; TAYLOR, K.C. Managing orchard floor vegetation in flood-irrigated citrus groves. HortTechnology, v.13, p.668-677, 2003.

Recebido em 2 de julho de 2008 e aprovado em 30 de dezembro de 2008 